\title{
The influence of fast waves and fluctuations on the evolution of the dynamics on the slow manifold
}

\author{
JARED P. WHITEHEA D ${ }^{1,2} \dagger$, BETH A. WINGATE ${ }^{1,2}$ \\ ${ }^{1}$ Department of Mathematics, Brigham Young University, Provo, UT, United States \\ ${ }^{2}$ Department of Mathematics, University of Exeter, Exeter, United Kingdom
}

(Received ?; revised ?; accepted ?. - To be entered by editorial office)

The effect of non-slow (typically fast) components of a rotating, stratified Boussinesq flow on the dynamics of the slow manifold is quantified using a decomposition that isolates the part of the flow living on the slow manifold. In this system there are three distinguished asymptotic limits with corresponding reduced equations, each defining a slow manifold. Each of these three distinct limits of rapid rotation, strong stratification, and simultaneously strong stratification and rapid rotation (quasi-geostrophy) are considered. Numerical simulations indicate that for the geometry considered (triply periodic) and the type of forcing applied, the fluctuations act as a conduit, moving energy onto the slow manifold. This decomposition clarifies how the energy is exchanged when either the stratification or the rotation is weak. In the quasi-geostrophic limit most of the energy transfer is between slow potential energy and slow kinetic energy, but the energetics due to the fluctuations are less clear. It is observed that the energy off the slow manifold in each case, equilibrates to a quasi-steady value.

\section{Key words:}

\section{Introduction}

Following the success of early numerical weather predictions based on Jule Charney's quasi-geostrophic approximation (see Charney (1948); Charney et al. (1950); Charney \& Phillips (1953)), there has been continued interest in reduced models and their relationship to the full system of equations for geophysical fluid dynamics. One reason for this interest is that reduced models avoid severe time step and spatial discretization restrictions required for accuracy and stability of the numerical algorithms for the full system. This yields improved utility over integrating the full system, at the cost of neglecting the accurate representation of the fast dynamics, long thought unimportant on the scales of interest.

Shortly after the breakthrough weather forecasts of the early 1950s, Charney (1955) considered the impact that such rapidly varying dynamics may have on the evolution of the so-called 'balanced' or slow state (quasi-geostrophy in this case). Charney supposed that if the influence of the fast dynamics (waves when considering the quasi-geostrophic limit) was insignificant in the initial phase, it would remain so afterward. Even so, the influence of waves on the mean flow and vice versa has been an active subject of re- 
search ever since, particularly when considering the initialization of the flow field (see Machenauer (1977); Baer (1977); Tribbia (1979); Baer \& Tribbia (1977); Leith (1980)).

Key to understanding the interplay between waves and mean flow in this sense is the definition of a slow manifold, originally initiated by Charney (1948); Leith (1980); Lorenz (1980). See Ford et al. (2000) for a formal definition as well as the references therein for the historical context. There followed some debate on whether such a manifold defined in the strictest sense, existed for the climate and weather systems (see Lorenz (1986); Lorenz \& Krishnamurthy (1987) for example). The contributions of E. N. Lorenz indicated that the existence of the slow manifold was dependent on the choice of model, although the methodology of using an asymptotic series in the hope of converging to a strictly invariant manifold was later questioned (Ford et al. 2000). In this vein Kreiss \& Lorenz (1994) showed that although the presence of fast waves in the initial state can be avoided, the existence of a slow manifold cannot always be guaranteed for the spatially continuous (partial differential equation) form of the system. Under assumptions of sufficient smoothness and within a finite time interval $[0, T]$ (where $T$ is independent of the rate of separation between the fast and slow dynamics), Kreiss \& Lorenz (1994) show that the spatially discrete version of the system will generate an exactly invariant slow manifold. The utility of such a result is questionable however, particularly due to the finite time interval restriction, and begs the question of whether such a result is indicative of the presence of a true manifold.

Further consideration of the finite (but possibly long) time invariance of the slow manifold is presented in Farge \& Sadourny (1989) where numerical simulations of the Saint-Venant shallow water equations indicate that the inertia-gravity waves do not directly influence the first order slow modes of the system. In contrast, Smith \& Waleffe (2002) show that forcing the small (presumably fast) scales of the weakly rotating, strongly stratified Boussinesq system (within a certain parameter regime of the rotation and stratification) generates large-scale, slow dynamics that are consistent with the vertically sheared horizontal flows predicted by Embid \& Majda (1998). Dewar \& Killworth (1995) considers a similar setting to demonstrate that while the fast waves (to $O(1)$ ) do not directly influence the dynamics on the slow manifold, the evolution of the slow dynamics will indirectly influence the fast waves as "the fast manifold variability "scatters" off the slow manifold structure". Ward \& Dewar (2010) further considers the effect that potential vorticity modes (on the slow manifold) will have on the fast inertia-gravity waves, clarifying that while no energy is transferred, the slow component of the flow will affect the distribution (both in space and frequency) of the fast waves. These investigations were motivated by the turbulence theory based predictions of Warn (1986); Warn \& Ménard (1986) that indicate that inertia-gravity waves will dominate the energetics and that these fast waves would continually emerge from solutions on or close to the slow manifold, something that appears to occur in the simulations reported by Smith \& Waleffe (2002). Such considerations are naturally in the context of a finite time interval, although as discussed in Section 4 the insight gained from Temam \& Wirosoetisno (2010) implies that similar statements may be made for long time dynamics as well.

To clarify the effect of the fast waves on the slow dynamics, Warn (1997); Ford et al. (2000) conclude that the slow manifold is not truly a manifold at all in the mathematical sense, but should be referred to as a 'fuzzy slow manifold' (as coined by Warn \& Ménard (1986)) or a 'slow quasimanifold'. While Warn (1997) indicates this nonexistence of the slow manifold to hold excepting very special cases, Ford et al. (2000) draws the analogy to Lighthill radiation although they also restrict the strength of the rotation to do so, as pointed out in Saujani \& Shepherd (2002). The non existence of an invariant slow manifold is further clarified in Vanneste \& Yavneh (2004) where an exact solution of 
the three-dimensional Boussinesq equations is shown to generate exponentially small inertia-gravity waves even under properly balanced initial conditions where no such waves are present. Vanneste (2013) provides a review of some of these ideas, emphasizing the role that the exponential asymptotics of Vanneste \& Yavneh (2004) have played in the understanding of spontaneous generation of fast waves by balanced or slow dynamical components of the flow.

As computational resources have increased and these concerns regarding the existence and veracity of a slow manifold have arisen, more complicated sets of equations have been used in order to capture all of the dynamics, both fast and slow. For example the fully compressible non hydrostatic system which includes all the fast waves removed by Charney's balanced equations is now in use for some operational forecast models (see Khairoutdinov et al. (2005); Staniforth \& Wood (2008) for two limited examples). With the advent of regional models and variable resolution grids, global models of atmospheric dynamics are now able to investigate physical regimes distinct from quasi-geostrophy and consider a wider range of phenomena than the large scale circulation. It is in this context that we consider the effect of slow dynamics on the full circulation of a system in which more than one limiting slow system is possible. We will investigate the effect that the fast part of the flow can have on the evolution of the slow component of the flow for each of these limiting regimes.

We investigate these effects via the rotating, stratified Boussinesq equations which yield three distinguished physically motivated reduced slow systems (Embid \& Majda 1998; Wingate et al. 2011). In the current work we do not consider any explicit asymptotic ansatz, but instead take the limiting systems derived via the separation of temporal scales in Embid \& Majda (1998); Wingate et al. (2011) as the $O(1)$ slow dynamics, and consider the decomposition of the flow into these components and fluctuations about these components, meaning that the fluctuations are really both the $O(1)$ fast components of the flow, and all $O(\epsilon)$ components as well. The slow systems outlined in Embid \& Majda (1998); Wingate et al. (2011) are rigorously justified as the limiting system of the full equations due to the theory of cancellation of oscillations as detailed in Klainerman \& Majda (1981); Schochet (1994). Essentially this theory relies on the fact that the fast operator is wave-generating only, and in the limit of an infinite separation of temporal scales, these fast waves cannot influence the dynamics of the rest of the flow (to $O(1)$ at least) because their net effect is averaged out over several wave periods.

For clarity of comparison with other work, we note that the rigorous justification of these limiting systems is distinct from the separation of temporal and spatial scale arguments used to derive the highly successful models of rapidly rotating Rayleigh Bénard convection (see Julien et al. (1998); Julien \& Knobloch (2007); Sprague et al. (2006); Julien et al. $(2006,2012 b)$ ). These investigations of rotating convection do not guarantee that there is no interaction between the $O(1)$ fast and $O(1)$ slow dynamics (in the terminology of Embid \& Majda (1998) the fast-fast-slow resonances do not necessarily vanish in the infinite limit) because the additional separation of spatial scales does not allow for the direct application of the theory of cancellation of oscillations (see Klainerman \& Majda (1981); Schochet $(1987,1994))$. This is dynamically a different situation than that studied in this paper, as the rapidly rotating limit studied here and in Wingate et al. (2011) is not even applicable to rotating convection where the limit of infinite rotation would yield a non-convective system in which the conductive state is asymptotically stable (see Chandrasekhar (1961) for example).

We define the slow manifold as the $O(1)$ part of the flow to which the full solution converges in the limit of infinite rotation/stratification, following the theory of cancellation of oscillations (see Klainerman \& Majda (1981); Schochet $(1987,1994)$ ). This definition 
is equivalent to that employed in previous work for the quasi-geostrophic limit, and allows us to incorporate the powerful theory of cancellation of oscillations into the other two limits studied here. In addition the slow manifold can be easily computed as the null space of the fast wave-generating operator. In this sense, we are allowing waves to be present on the slow manifold, as generated by a 'slow' wave-generating operator. For example, in the limit of rapid rotation but weak stratification, the slow manifold will retain waves generated by the stratification of the flow, but those generated by the rotation will be contained in the fluctuating part of the flow as the rotation is the limiting operator in this case. Thus, the decomposition into the slow manifold and fluctuations about it is not necessarily a consideration of the separation between the mean flow and waves.

To consider how the energy flows on and off the 'slow manifold', we decompose the full Boussinesq flow into the component living on the slow manifold (that part that lives in the null space of the dominating wave-generating operator) and fluctuations about the slow manifold (both the $O(1)$ fast waves and all $O(\epsilon)$ components of the flow). This is done for each of the three distinct limiting regimes for this system: rapid rotation with weak stratification (Wingate et al. 2011), and strong stratification with either weak or rapid rotation (Embid \& Majda 1998). As already mentioned, we make no asymptotic ansatz on the system, but instead consider the reduced systems previously justified via the theory of cancellation of oscillations as described in Klainerman \& Majda (1981); Majda (1984); Schochet $(1987,1994)$. This allows for the complete separation of fast and slow dynamics in the infinite limit (to $O(1)$ for finite values of the relevant parameters). The current decomposition leads to new evolution equations for the energy on and off the $O$ (1) slow manifol. High resolution numerical simulations indicate that the fluctuating part of the flow acts as a conduit to move energy onto the slow manifold in the form of kinetic slow energy. This result is robust when either the stratification or rotation is weak (not quasi-geostrophic). The movement of energy in the quasi-geostrophic regime is more complicated. From quasi-geostrophic theory, we expect the energy movement to take place primarily on the slow manifold, i.e. from available slow potential energy (as injected via our choice of forcing) into the slow kinetic energy. In our simulations the most significant movement of energy occurs through this mechanism so deducing clear statements about the role of the fluctuations relative to the forcing is not clear nor instructive. This is the only limit in which energy can be exchanged from potential to kinetic and vice versa while remaining on the slow manifold.

In the following section we introduce the rotating, stratified Boussinesq equations in the context of the method of multiple scales and for completeness review the limiting slow dynamics for each of the three distinct limits as originally derived in Embid \& Majda (1998); Wingate et al. (2011). After separating the flow into $O(1)$ slow components and fluctuations about this slow manifold, we determine the evolution of the flow on the slow manifold, including possible sources from the non-slow parts of the flow. In Section 3 we use direct numerical simulations in this context to evaluate the influence of the fast part of the flow (everything not living on the $O(1)$ slow manifold) on dynamics on the slow manifold for each of the three distinct limits. Section 4 contains conclusions that can be drawn from the simulations as well as suggestions for further work. 


\section{The Boussinesq system in the presence of rapid rotation and/or strong stratification}

2.1. Dynamics of the full system and an introduction to the separation of time scales

The rotating, stratified Boussinesq equations, with a linear stably stratifying background buoyancy profile, are

$$
\begin{gathered}
\frac{\partial \boldsymbol{v}}{\partial t}+\boldsymbol{v} \cdot \nabla \boldsymbol{v}+f \hat{z} \times \boldsymbol{v}+\frac{1}{\rho_{0}} \rho g \hat{z}+\frac{1}{\rho_{0}} \nabla p=\nu \Delta \boldsymbol{v}, \\
\nabla \cdot \boldsymbol{v}=0 \\
\frac{\partial \rho}{\partial t}+\boldsymbol{v} \cdot \nabla \rho-b w=\kappa \Delta \rho,
\end{gathered}
$$

where $\boldsymbol{v}$ is the three dimensional velocity, and $\rho$ is the buoyancy variable (density). The Coriolis force (indicative of the strength of the rotation) is $f, \rho_{0}$ is a reference density, $g$ is the force of gravitational acceleration, $\nu$ is the kinematic viscosity, $b$ is the strength of the underlying density stratification, and $\kappa$ is the coefficient of diffusivity for the buoyancy. In order to make the system tractable, we consider a length scale $L$ and velocity scale $U$ with corresponding advective time scale $L / U$. In addition we prescribe the buoyancy fluctuation scale $b U / N$ where $N=\left(g b / \rho_{0}\right)^{1 / 2}$ is the Brunt-Väisälä frequency. This yields the non-dimensional equations:

$$
\begin{gathered}
\frac{\partial \boldsymbol{v}}{\partial t}+\boldsymbol{v} \cdot \nabla \boldsymbol{v}+\frac{1}{R o} \hat{z} \times \boldsymbol{v}+E u \nabla p+\frac{1}{F r} \rho \hat{z}=\frac{1}{R e} \Delta \boldsymbol{v}, \\
\nabla \cdot \boldsymbol{v}=0 \\
\frac{\partial \rho}{\partial t}+\boldsymbol{v} \cdot \nabla \rho-\frac{1}{F r} w=\frac{1}{R e \operatorname{Pr}} \Delta \rho,
\end{gathered}
$$

where $R o=\frac{U}{f L}$ measures the relative advective time scale to the time scale induced by rotation, $E u=\frac{P}{\rho U^{2}}$ rescales the pressure, $R e=\frac{U L}{\nu}$ is the traditional Reynold's number measuring the relative effect of inertia to viscous dissipation, $\operatorname{Pr}=\frac{\nu}{\kappa}$ evaluates the relative effect of dissipation of momentum to dissipation of density, and $\mathrm{Fr}=\frac{U}{N L}$ quantifies the differences between advective and buoyancy fluctuation time-scales. We will not differentiate between the dimensional and non-dimensional velocity and buoyancy, and wherever such a distinction becomes necessary, we will highlight it explicitly in the body of the paper.

In the following we follow the formulation of Embid \& Majda (1998) and write the dependent variables in vector format as

$$
\boldsymbol{u}=\left(\begin{array}{c}
\boldsymbol{v} \\
\rho
\end{array}\right)
$$

and rewrite the momentum equation in its nonlocal form (solving explicitly for the pressure)

$$
\frac{\partial \boldsymbol{v}}{\partial t}+\boldsymbol{v} \cdot \nabla \boldsymbol{v}+\frac{1}{R o} \hat{z} \times \boldsymbol{v}+\nabla \Delta^{-1}\left(\frac{1}{R o} \hat{z} \cdot \boldsymbol{\omega}-\frac{1}{F r} \frac{\partial \rho}{\partial z}-\nabla \cdot(\boldsymbol{v} \cdot \nabla \boldsymbol{v})\right)+\frac{1}{F r} \rho \hat{z}=\frac{1}{R e} \Delta \boldsymbol{v},
$$

where $\boldsymbol{\omega}=\nabla \times \boldsymbol{v}$ is the vector-valued relative vorticity.

Following (Embid \& Majda 1996, 1998; Wingate et al. 2011) the full system is rewritten 
as

$$
\frac{\partial \boldsymbol{u}}{\partial t}+\frac{1}{R o} L_{R o} \boldsymbol{u}+\frac{1}{F r} L_{F r} \boldsymbol{u}+B(\boldsymbol{u}, \boldsymbol{u})=D \boldsymbol{u},
$$

where

$$
\begin{aligned}
& L_{R o} \boldsymbol{u}=\left(\begin{array}{c}
\hat{z} \times \boldsymbol{v}_{H}+\nabla_{H} \Delta^{-1} \hat{z} \cdot \boldsymbol{\omega} \\
\partial_{z} \Delta^{-1} \hat{z} \cdot \boldsymbol{\omega} \\
0
\end{array}\right), \\
& L_{F r} \boldsymbol{u}=\left(\begin{array}{c}
-\nabla_{H} \Delta^{-1} \rho_{z} \\
-\Delta^{-1} \rho_{z z}+\rho \\
-w
\end{array}\right) \text {, } \\
& B(\boldsymbol{u}, \boldsymbol{u})=\left(\begin{array}{c}
\boldsymbol{v} \cdot \nabla \boldsymbol{v}-\nabla \Delta^{-1} \nabla \cdot(\boldsymbol{v} \cdot \nabla \boldsymbol{v}) \\
\boldsymbol{v} \cdot \nabla \rho
\end{array}\right), \\
& D \boldsymbol{u}=\frac{1}{R e}\left(\begin{array}{c}
\Delta \boldsymbol{v} \\
\frac{1}{\operatorname{Pr}} \Delta \rho
\end{array}\right) .
\end{aligned}
$$

Embid \& Majda (1998); Wingate et al. (2011) consider the limits of $\mathrm{Fr} \rightarrow 0$ and/or $R o \rightarrow 0$, deriving the slow equations for each of these three limits, relying on the theory of cancellation of fast oscillations developed by Klainerman \& Majda (1981); Schochet (1994). There is a corresponding projection operator $P_{\alpha}$ onto the null space of the fast linear operator for each limit that projects the flow onto the relevant slow manifold. In order to understand how the flow approaches and interacts with the slow manifold we decompose the full solution as

$$
\boldsymbol{u}=\boldsymbol{u}^{\alpha}+\boldsymbol{u}_{\alpha}^{\prime}
$$

where $\alpha$ represents the $R o \rightarrow 0, F r \rightarrow 0$ or the simultaneous limit of both ( $Q G$ for quasi-geostrophy), and where

$$
P_{\alpha} \boldsymbol{u}^{\alpha}=\boldsymbol{u}^{\alpha}, \quad P_{\alpha} \boldsymbol{u}_{\alpha}^{\prime}=0 .
$$

This decomposition is used to find evolution equations for the components of the flow (and the corresponding energy) on and off the slow manifold. This decomposition is independent of $\mathrm{Fr}$ and $R o$ regardless of which limiting system is under consideration, so in Section 3 we look at moderate to small values of the relevant parameter. In addition we emphasize that this decomposition does not presuppose any asymptotic ansatz or separation in scales, but simply separates the flow into that part living on the slow manifold (as defined as the null space of the dominant wave-generating operator) and the fluctuations off the slow manifold.

\subsection{Rapid rotation in the presence of weak stratification}

Here we consider the dynamics in the presence of rapid rotation with weak stratification. Parametrically this is where $R o \rightarrow 0$ and $F r=O(1)$. For our purposes, the slow manifold in this limit is defined as the null space of $L_{R o}$ described in equation (2.11).

As this limit has not been studied as much as the other two limits, we provide some examples of physically relevant circumstance where this may occur. These include non hydrostatic baroclinic and symmetric instability (see Stone (1966); Stone et al. (1969); Stone $(1971,1972)$ ), non hydrostatic dynamics in the Mediterranean sea (see Van Haren \& Millot $(2005 a, b))$ where it is observed that $N=0 \pm .4 f(2.5<f / N<\infty)$ and Van Haren \& Millot $(2005 a, b)$ argue that the dynamics in these particular regions are driven by both weak stratification and rotation in the horizontal. Another region of the world 
where strong rotation and weak stratification have been observed is in the deep Arctic Ocean. Measurements in the Beaufort Gyre by Timmermans et al. (2007); Timmermans (2010) show $f / N \approx 2$ above 2600 meters and $f / N \approx \infty$ between the depths of 2600 and $3600 \mathrm{~m}$. Weak stratification in the deep ocean at high latitudes has been noted for the North Atlantic and North Pacific in Emery et al. (1984) where they compute mean profiles of density and Brunt-Väisäla frequency; in the deep waters of the Arctic Ocean by Jones et al. (1995); and in the Southern Ocean by Heywood et al. (2002). Another situation where rotation is important, but where the stratification is unstable and actually provides the forcing, is rotating convection, although the limiting system in that case is trivially purely conductive (see Julien et $a l$. $(2012 b, a)$ and references therein as well as Ahlers et al. (2009); Liu \& Ecke (2009); Zhong \& Ahlers (2010); King et al. (2012) for experimental work on the same).

The projection operator for the null space of $L_{R o}$ was computed exactly as equation (3.17) in Wingate et al. (2011) and is repeated here for completeness:

$$
P_{R o} \boldsymbol{u}=\left(\begin{array}{c}
\left\langle\boldsymbol{v}_{H}\right\rangle_{z}-\nabla_{H} \Delta_{H}^{-1}\left(\nabla_{H} \cdot\left\langle\boldsymbol{v}_{H}\right\rangle_{z}\right) \\
\langle w\rangle_{z} \\
\rho
\end{array}\right),
$$

where $\langle\cdot\rangle_{z}$ indicates the vertical average. This leads to the evolution equation of the flow on the slow manifold:

$$
\begin{gathered}
\frac{\partial \boldsymbol{v}_{H}^{R o}}{\partial t}+\boldsymbol{v}_{H}^{R o} \cdot \nabla_{H} \boldsymbol{v}_{H}^{R o}-\nabla_{H} \Delta_{H}^{-1}\left(\nabla_{H} \cdot\left(\boldsymbol{v}_{H}^{R o} \cdot \nabla_{H} \boldsymbol{v}_{H}^{R o}\right)\right)-\frac{1}{R e} \Delta_{H} \boldsymbol{v}_{H}^{R o} \\
=-\left(1-\nabla_{H} \Delta_{H}^{-1} \nabla_{H} \cdot\right)\left\langle\left\{\boldsymbol{v}^{\prime} \cdot \nabla \boldsymbol{v}^{\prime}\right\}_{H}\right\rangle_{z}, \\
\nabla_{H} \cdot \boldsymbol{v}_{H}^{R o}=0, \\
\frac{\partial w^{R o}}{\partial t}+\boldsymbol{v}_{H}^{R o} \cdot \nabla_{H} w^{R o}+\frac{1}{F r}\langle\rho\rangle_{z}-\frac{1}{R e} \Delta_{H} w^{R o}=-\left\langle\boldsymbol{v}^{\prime} \cdot \nabla w^{\prime}\right\rangle_{z}, \\
\frac{\partial \rho}{\partial t}+\boldsymbol{v}^{R o} \cdot \nabla \rho-\frac{1}{F r} w^{R o}-\frac{1}{R e \operatorname{Pr}} \Delta \rho=-\boldsymbol{v}^{\prime} \cdot \nabla \rho+\frac{1}{F r} w^{\prime},
\end{gathered}
$$

where we have dropped the subscript reference to the rapid rotation limit in the primed variables to avoid unnecessarily complex notation. When we are concerned with one of the other limits $\mathrm{Fr} \rightarrow 0$, we will clearly indicate this in the text. The system (2.18)-(2.21) reduces to the slow equations derived in Wingate et al. (2011) when the fluctuations are omitted, i.e. the primed variables vanish.

These evolution equations lead to the evolution for the volume averaged energy on the slow manifold as

$$
\begin{gathered}
\frac{1}{2} \frac{d}{d t}\left\|\boldsymbol{v}_{H}^{R o}\right\|_{2}^{2}+\frac{1}{R e}\left\|\nabla_{H} \boldsymbol{v}_{H}^{R o}\right\|_{2}^{2}=-\int_{A} \boldsymbol{v}_{H}^{R o} \cdot\left\langle\left\{\boldsymbol{v}^{\prime} \cdot \nabla \boldsymbol{v}^{\prime}\right\}_{H}\right\rangle_{z} d A, \\
\frac{1}{2} \frac{d}{d t}\left\|w^{R o}\right\|_{2}^{2}+\frac{1}{R e}\left\|\nabla_{H} w^{R o}\right\|_{2}^{2}=-\frac{1}{F r} \int_{A} w^{R o}\langle\rho\rangle_{z} d A-\int_{A} w^{R o}\left\langle\boldsymbol{v}_{H}^{\prime} \cdot \nabla_{H} w^{\prime}\right\rangle_{z} d A, \\
\frac{1}{2} \frac{d}{d t}\|\rho\|_{2}^{2}+\frac{1}{R e \operatorname{Pr}}\|\nabla \rho\|_{2}^{2}=\frac{1}{F r} \int_{V} w^{R o} \rho d V+\frac{1}{F r} \int_{V} w^{\prime} \rho d V .
\end{gathered}
$$

This indicates that the only way that potential energy can move to the component of horizontal kinetic energy on the slow manifold, is through the effect of non-slow dynamics (the primed variables). Therefore, adding a force to the potential energy immediately gives a measure of how the fluctuations interact with the slow manifold. 


\subsection{Strong stratification in the presence of weak rotation}

This is the regime for which $\mathrm{Fr} \rightarrow 0$ and $R o=O(1)$. Here the slow manifold is defined as the null space of $L_{F r}$ described by equation (2.12). Some examples of this occurring include the strong stratification range of the nocturnal atmospheric boundary layer Coulter (1990); Mahrt et al. (1998); Conangla et al. (2008); Kumar et al. (2012), atmospheric moist stratified convection Sukhatme et al. (2012), observations of the tidal bottom boundary layer Werner et al. (2003), and some fundamental considerations in geophysical fluid dynamics Smith \& Waleffe (2002); Majda \& Grote (1997); Lelong \& Riley (1991); Riley \& Lelong (2000).

The projection operator for the null space of $L_{F r}$ was computed exactly in Embid \& Majda (1998):

$$
P_{F r} \boldsymbol{u}=\left(\begin{array}{c}
\boldsymbol{v}_{H}-\nabla_{H} \Delta_{H}^{-1}\left(\nabla_{H} \cdot \boldsymbol{v}_{H}\right) \\
0 \\
\langle\rho\rangle_{H}
\end{array}\right),
$$

where $\langle\cdot\rangle_{H}$ is the horizontal average. This leads to the evolution of the components of the flow on the slow manifold as:

$$
\begin{gathered}
\frac{\partial \boldsymbol{v}_{H}^{F r}}{\partial t}+\boldsymbol{v}_{H}^{F r} \cdot \nabla_{H} \boldsymbol{v}_{H}^{F r}+\frac{1}{R o} \hat{z} \times \boldsymbol{v}_{H}^{F r}+\nabla_{H} \Delta_{H}^{-1}\left(\frac{1}{R o} \omega^{F r}-\nabla_{H} \cdot\left(\boldsymbol{v}_{H}^{F r} \cdot \nabla_{H} \boldsymbol{v}_{H}^{F r}\right)\right) \\
=\frac{1}{R e} \Delta \boldsymbol{v}_{H}^{F r}-\left(1-\nabla_{H} \Delta_{H}^{-1} \Delta_{H} \cdot\right)\left\{\boldsymbol{v}^{\prime} \cdot \nabla \boldsymbol{v}^{\prime}\right\}_{H} \\
\frac{\partial \rho^{F r}}{\partial t}=\frac{1}{R e \operatorname{Pr}} \frac{\partial^{2} \rho^{F r}}{\partial z^{2}}-\left\langle\boldsymbol{v}^{\prime} \cdot \nabla \rho^{\prime}\right\rangle_{H}
\end{gathered}
$$

where once again the slow reduced equations of Embid \& Majda (1998) are recovered when the inertia-gravity waves (primed variables) vanish. The energy on the slow manifold then evolves according to:

$$
\begin{aligned}
& \frac{1}{2} \frac{d}{d t}\left\|\boldsymbol{v}_{H}^{F r}\right\|_{2}^{2}+\frac{1}{R e}\left\|\nabla \boldsymbol{v}_{H}^{F r}\right\|_{2}^{2}=-\int_{V} \boldsymbol{v}_{H}^{F r} \cdot\left\{\boldsymbol{v}^{\prime} \cdot \nabla \boldsymbol{v}^{\prime}\right\}_{H} d V \\
& \frac{1}{2} \frac{d}{d t}\left\|\rho^{F r}\right\|_{2}^{2}+\frac{1}{R e \operatorname{Pr}}\left\|\frac{\partial \rho^{F r}}{\partial z}\right\|_{2}^{2}=-\int \rho^{F r}\left\langle\boldsymbol{v}^{\prime} \cdot \nabla \rho^{\prime}\right\rangle_{H} d z .
\end{aligned}
$$

Again there is no exchange between slow kinetic and slow potential energy. Said another way, the exchange between slow kinetic and slow potential energy in this limit relies on the fluctuating dynamics.

\subsection{Rapid rotation and simultaneously strong stratification}

This is the quasi-geostrophic limit where $R o, F r \rightarrow 0$ and $F r / R o=f / N$ is finite. In this limit the slow manifold is the null space of $L_{F r}+B u L_{R o}$ where $B u=F r / R o$ is the Burger number. These linear operators are described by equations (2.11) and (2.12). This limit is well studied in the atmosphere and oceans as indicated by the descriptions in Pedlosky (1987); Vallis (2006). As two examples, quasi-geostrophic turbulence is of fundamental interest to understanding the evolution of large scale dynamics (Charney 1971) and this system provides one of the simplest models which inherits the fundamental dynamics responsible for baroclinic instability (Charney 1947; Eady 1949).

Although Embid \& Majda (1998) consider this limit and derive the evolution of the flow on the slow manifold in terms of the potential vorticity, they do not explicitly 
compute the projection operator onto the null space of $L_{F r}+B u L_{R o}$ in their paper. This calculation is performed in the appendix in this paper to demonstrate how the projection operator for a more general system could be computed, and is given by:

$$
P_{Q G} \boldsymbol{u}=\left(\begin{array}{c}
\boldsymbol{v}_{H}-B u^{2} \Delta_{Q G}^{-1} \frac{\partial^{2} \boldsymbol{v}_{H}}{\partial z^{2}}-\Delta_{Q G}^{-1}\left(\nabla_{H}\left(\nabla_{H} \cdot \boldsymbol{v}_{H}\right)+B u \nabla_{H} \times\left(\hat{z} \frac{\partial \rho}{\partial z}\right)\right) \\
0 \\
\rho-B u \Delta_{Q G}^{-1}\left(\frac{\partial}{\partial z}\left(\nabla_{H} \times \boldsymbol{v}_{H}\right)\right)-\Delta_{Q G}^{-1} \Delta_{H} \rho
\end{array}\right)
$$

where $\Delta_{Q G}=\Delta_{H}+B u^{2} \frac{\partial^{2}}{\partial z^{2}}$ is the modified QG Laplacian operator. This leads to new evolution equations for the slow dynamics in terms of the velocity and density fields (as opposed to potential vorticity which is traditionally the variable of choice in this limit)

$$
\begin{gathered}
\frac{\partial \boldsymbol{v}_{H}^{Q G}}{\partial t}+\boldsymbol{v}_{H}^{Q G} \cdot \nabla_{H} \boldsymbol{v}_{H}^{Q G}-B u^{2} \Delta_{Q G}^{-1} \frac{\partial^{2}}{\partial z^{2}}\left(\boldsymbol{v}_{H}^{Q G} \cdot \nabla \boldsymbol{v}_{H}^{Q G}\right) \\
-\Delta_{Q G}^{-1}\left(\nabla_{H}\left(\nabla_{H} \cdot \boldsymbol{v}_{H}^{Q G} \cdot \nabla_{H} \boldsymbol{v}_{H}^{Q G}\right)-B u \nabla_{H} \times\left(\hat{z} \frac{\partial}{\partial z}\left\{\boldsymbol{v}_{H}^{Q G} \cdot \nabla \rho^{Q G}\right\}\right)\right)-\frac{1}{R e} \Delta \boldsymbol{v}_{H}^{Q G} \\
=-\left\{\boldsymbol{v}^{\prime} \cdot \nabla \boldsymbol{v}^{\prime}\right\}_{H}+\Delta_{Q G}^{-1}\left(\nabla_{H}\left(\nabla_{H} \cdot\left\{\boldsymbol{v}^{\prime} \cdot \nabla \boldsymbol{v}^{\prime}\right\}_{H}\right)-B u \nabla_{H} \times\left(\hat{z} \frac{\partial}{\partial z}\left\{\boldsymbol{v}^{\prime} \cdot \nabla \rho^{\prime}\right\}\right)\right) \\
+B u^{2} \Delta_{Q G}^{-1} \frac{\partial^{2}}{\partial z^{2}}\left\{\boldsymbol{v}^{\prime} \cdot \nabla \boldsymbol{v}^{\prime}\right\}_{H} \\
\frac{\partial \rho^{Q G}}{\partial t}+\boldsymbol{v}_{H}^{Q G} \cdot \nabla \rho^{Q G}-B u \Delta_{Q G}^{-1} \frac{\partial}{\partial z}\left(\boldsymbol{v}_{H}^{Q G} \cdot \nabla_{H} \omega^{Q G}\right)-\Delta_{Q G}^{-1} \Delta_{H}\left(\boldsymbol{v}_{H}^{Q G} \cdot \nabla \rho^{Q G}\right) \\
-\frac{1}{R e \operatorname{Pr}} \Delta \rho^{Q G}=-\boldsymbol{v}^{\prime} \cdot \nabla \rho^{\prime}+B u \Delta_{Q G}^{-1} \frac{\partial}{\partial z}\left(\nabla_{H} \times\left\{\boldsymbol{v}^{\prime} \cdot \nabla \boldsymbol{v}^{\prime}\right\}_{H}\right)+\Delta_{Q G}^{-1} \Delta_{H}\left(\boldsymbol{v}^{\prime} \cdot \nabla \rho^{\prime}\right) .
\end{gathered}
$$

When the non-slow components of the flow vanish and the potential vorticity is defined as in Embid \& Majda (1998) this reduces to advection of potential vorticity as is well known for this limit. Though the potential vorticity equation would be an interesting quantity to examine using this technique, we are attempting to look at the flow of energy in all three limits so we leave the discussion of potential vorticity and potential enstrophy 
for future work. The energy on the slow manifold evolves according to

$$
\begin{gathered}
\frac{1}{2} \frac{d}{d t}\left\|\boldsymbol{v}_{H}^{Q G}\right\|_{2}^{2}+\frac{1}{R e}\left\|\nabla \boldsymbol{v}_{H}^{Q G}\right\|_{2}^{2}= \\
-B u \int \boldsymbol{v}_{H}^{Q G} \cdot \Delta_{Q G}^{-1}\left[\nabla_{H} \times\left(\hat{z} \frac{\partial}{\partial z}\left[\boldsymbol{v}_{H}^{Q G} \cdot \nabla \rho^{Q G}\right]\right)\right] \\
-\int \boldsymbol{v}_{H}^{Q G} \cdot\left(1-B u^{2} \Delta_{Q G}^{-1} \frac{\partial^{2}}{\partial z^{2}}\right)\left\{\boldsymbol{v}^{\prime} \cdot \nabla \boldsymbol{v}^{\prime}\right\}_{H} \\
-B u \int \boldsymbol{v}_{H}^{Q G} \cdot \Delta_{Q G}^{-1}\left[\nabla_{H} \times\left(\hat{z} \frac{\partial}{\partial z}\left\{\boldsymbol{v} \cdot \nabla \rho^{\prime}\right\}\right)\right] \\
\frac{1}{2} \frac{d}{d t}\left\|\rho^{Q G}\right\|_{2}^{2}+\frac{1}{R e \operatorname{Pr}}\|\nabla \rho\|_{2}^{2}= \\
B u \int \rho^{Q G} \Delta_{Q G}^{-1} \frac{\partial}{\partial z}\left(\boldsymbol{v}_{H}^{Q G} \cdot \nabla_{H} \omega^{Q G}\right) \\
-\int \rho^{Q G}\left(1-\Delta_{Q G}^{-1} \Delta_{H}\right) \boldsymbol{v}^{\prime} \cdot \nabla \rho^{\prime} \\
+B u \int \rho^{Q G} \Delta_{Q G}^{-1} \frac{\partial}{\partial z}\left(\nabla_{H} \times\left\{\boldsymbol{v} \cdot \nabla \boldsymbol{v}^{\prime}\right\}_{H}\right) .
\end{gathered}
$$

The first term on the right hand side of both (2.33) and (2.34) describes the direct transfer of kinetic to potential energy on the slow manifold. This is known to occur during baroclinic instability as described by Eady (1949) and explained further in Pedlosky (1987). Though we expect there to be slow transfers between potential and kinetic in the QG limit, it is interesting that there are no such direct transfer terms in the other two limits. The remaining four integral quantities are indicative of the transfer onto or off the slow manifold. We consider these four integrals separately to ascertain the influence of the fluctuations on the slow kinetic and potential energy.

\section{Numerical Simulations}

\subsection{Description of the Numerical Algorithm}

For all of the simulations presented in this section, we use the triply periodic, pseudospectral LANL/Sandia Direct Numerical Simulation (DNS) code. Details of the code, along with evidence of its scalability are detailed in Wingate et al. (2011). The dimensional equations (2.1)-(2.3) are integrated via a pseudo-spectral method on a $[0,1]^{3}$ triply periodic box with a Runge-Kutta 4 explicit time-stepping.

We consider a configuration of our numerical experiments similar to that used in Smith \& Waleffe (2002); Wingate et al. (2011). Smith \& Waleffe (2002) numerically investigates different parameter ranges of these equations and shows that for strongly stratified flows the slow large scales generated by the small scale forcing represent vertically sheared horizontal flows as predicted by Embid \& Majda (1998), corresponding to the limit we discuss in Section 3.3. Smith \& Waleffe (2002)used the spectra of the flow to deduce the effects of small scales on the large. In this work we also use a stochastic forcing with unit standard deviation and centered about wave number $k_{f}$, but the wave number we choose depends on which limit we are considering. The reason for different choices of the forcing scale can be understood by considering the relationship of the Rossby deformation radius 
to the forcing scale, $L_{d}$,

$$
L_{d}=\frac{N}{f} L_{f} \quad \text { or } \quad k_{d}=\frac{f}{N} k_{f},
$$

where $k_{f}$ is the peak wave number of the forcing and $k_{d}$ is the wave number of the deformation radius. This equation shows that the important horizontal length scales (where the energy will move toward) described by $k_{d}$ decrease with increasing rotation rate assuming $N$ is fixed. Therefore we choose low wave number $k_{f}=3(2 \pi)$ forcing (the $2 \pi$ is because the domain for the simulations is a $[0,1]^{3}$ box) for fast rotation and weak stratification, and high wave number $k_{f}=24(2 \pi)$ for strong stratification and weak rotation. The same small scale $k_{f}=24(2 \pi)$ is selected for the quasi-geostrophic limit, as it is well established that this limit will produce an inverse cascade similar to that observed in two-dimensional turbulence (see Vallis (2006)).

An explicit formula for the forcing spectrum for this geometry is given as eqn (4.3) in Wingate et al. (2011). In all of the simulations reported here, the forcing is applied to the buoyancy equation only. The motivation for forcing the buoyancy is that in the distinct limits when either rotation or stratification is weak, the potential energy is decoupled from the kinetic energy on the slow manifold. We do not expect such an observation to hold for the simulations reported in this paper because the rotation and stratification are finite, however the separation between slow kinetic and potential energy will indicate the validity of considering the dynamics on the slow manifold even when the infinite limit is not reached. We acknowledge that the potential and kinetic energy is not decoupled for the QG limit, but we maintain the same type of forcing for all three limiting cases in order to maintain some level of consistency in the comparisons that follow.

The code is run in a dimensional configuration wherein the Coriolis force $f$ and BruntVaisala frequency $N$ are prescribed as well as the forcing wavenumber $k_{f}$. The forcing with $k_{f}$ and energy input rate $\epsilon_{f}\left(\epsilon_{f}=1\right.$ in all of these simulations) yields an advective (eddy turnover) time-scale of

$$
\tau=\left(\epsilon_{f}\left(k_{f}\right)^{2}\right)^{-1 / 3}
$$

where the $2 \pi$ is necessary because the code is run on the $[0,1]^{3}$ box. From this the effective Rossby and Froude numbers are easily computed as

$$
\operatorname{Ro}=\frac{\tau}{f}, \quad \text { and } \quad F r=\frac{\tau}{N},
$$

and the velocity scale is

$$
U=\left(\epsilon_{f}\left(k_{f}\right)^{-1}\right)^{1 / 3}
$$

This allows for direct non-dimensionalization of the corresponding output.

For clarity we note that the same hyper viscosity (first introduced in Chasnov (1994)) and used in Smith \& Waleffe (1999); Wingate et al. (2011) is utilized. The use of such a modeled dissipation allows us to investigate a far greater range of scales than the standard Laplacian viscosity. In addition, stronger values of the rotation and stratification can be considered here due to higher resolution $\left(512^{3}\right.$ for all reported simulations).

\subsection{Rapid rotation in the presence of weak stratification}

The evolution of the horizontal kinetic and potential energy is shown in Fig. 1. Initially the flow evolves as one would expect being forced through the density, the potential energy grows rapidly and there is a spin-up period in which the slow horizontal kinetic 

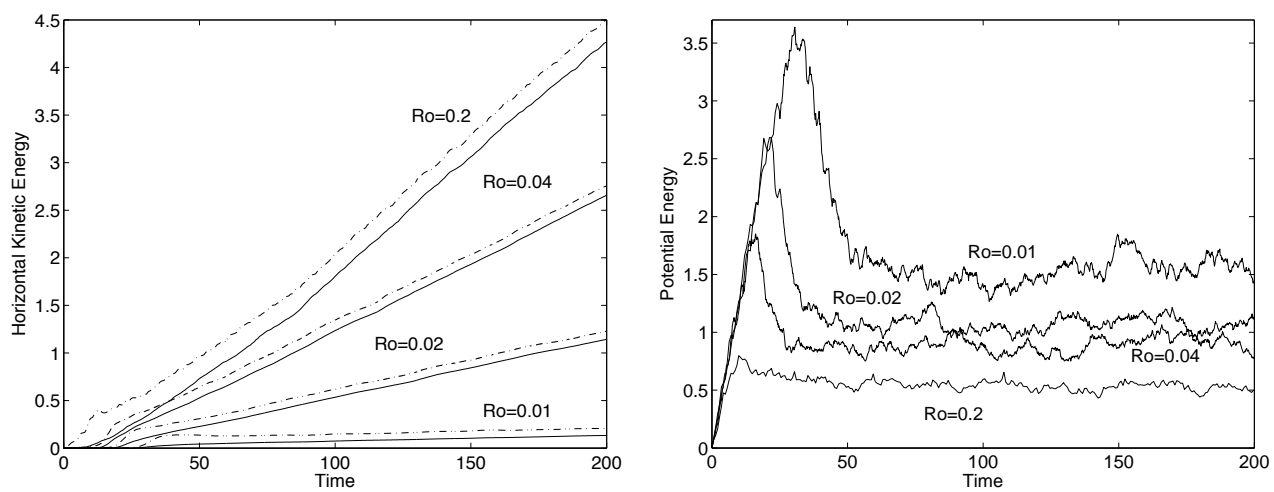

FiguRE 1. The evolution of the horizontal kinetic energy (left) and potential energy (right) for a handful of the rapidly rotating and weakly stratified simulations. The dashed lines in the left plot indicate the full horizontal kinetic energy while the solid lines represent the development of the horizontal kinetic energy on the slow manifold.

energy (solid lines in the left hand plot) remains dormant. Following this spin-up phase, the potential energy equilibrates to a quasi-steady value, and the slow horizontal kinetic energy grows linearly in time, while the non-slow horizontal kinetic energy also reaches a quasi-steady value. This indicates that after the initial (Ro dependent) spin-up phase the energy is transferred from the potential where it is inserted via the forcing, to the slow horizontal kinetic. Recalling (2.22)-(2.24), this can only occur via the exchange between the potential energy and the non-slow components of the flow, i.e. the flow off of the slow manifold.

To quantify these observations, Fig. 2 displays the time evolution of the two integral quantities from (2.22) and (2.23) that show the effect of the fluctuations on the evolution of the slow manifold. For the forcing used in these simulations, and for the rotation strong enough $(R o \leqslant 0.2)$ the sign of these exchange terms remains constant, indicating that the non-slow components of the flow have a consistent influence on the slow dynamics.

Although Fig. 3 only shows the correlation of the density and vertical velocity variables for $R o=0.01$, similar results hold for the other values of $R o$. The correlation of density with the fluctuating vertical velocity appears to act as a sink for potential energy (as seen by the second plot in Fig. 3), whereas the correlation with the slow vertical velocity appears to oscillate indicating a rather ambiguous exchange between the potential and slow vertical kinetic energy. Using the observations gained from Figs. 2 and 3 and returning to $(2.22)$ and $(2.23)$ we see that the particular sign of these integral exchanges imply that the fluctuating component of the flow acts as a sink for slow vertical kinetic energy as well as the potential energy, and as a source for slow horizontal kinetic energy. In summary, after a spin-up time, our simulations show that the energy injected into the potential is moved to the vertical kinetic and then universally transferred to the non-slow fluctuating component of the flow and then to the slow horizontal kinetic.

For these simulations then, we observe that the non-slow part of the flow does not grow in time. This is particularly true for the horizontal momentum as indicated by the near constant difference between the dashed and solid lines in the left plot of Fig. 1 (although not shown a similar statement can be made for the vertical kinetic energy as well). Instead the part of the flow off the slow manifold acts purely as a conduit to transfer energy onto the slow manifold in the form of slow horizontal kinetic energy. 

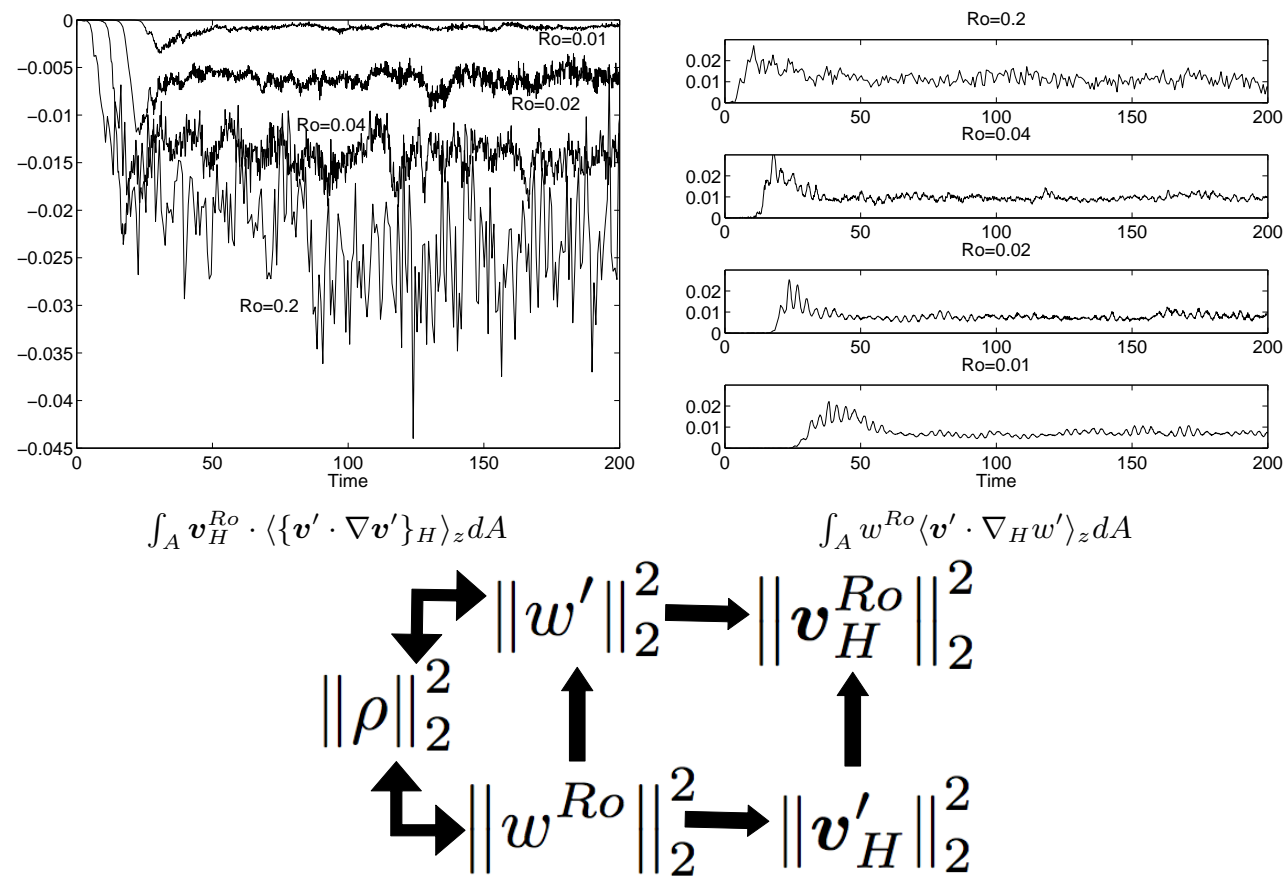

FiguRE 2. The exchange between the non-slow components of the flow and the slow horizontal kinetic energy (left) and slow vertical kinetic energy (right) for a handful of the rapidly rotating, weakly stratified simulations. Each of these quantities has a definite sign (for all time $t$ ) for $R o \leqslant 0.2$. A schematic of the movement of the energy is also shown.

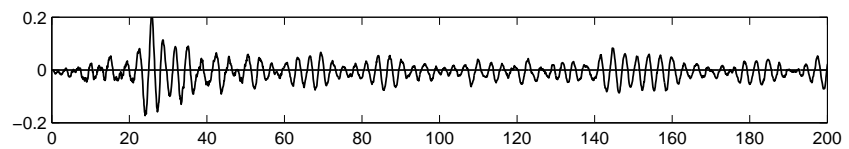

$$
\frac{1}{F r} \int_{V} w^{R o} \rho d V
$$

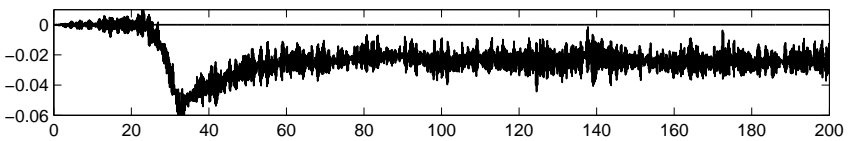

$$
\frac{1}{F r} \int_{V} w^{\prime} \rho d V
$$

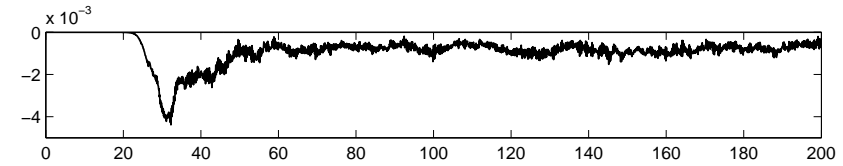

$$
\int_{A} \boldsymbol{v}_{H}^{R o} \cdot\left\langle\left\{\boldsymbol{v}^{\prime} \cdot \nabla \boldsymbol{v}^{\prime}\right\}_{H}\right\rangle_{z} d A
$$

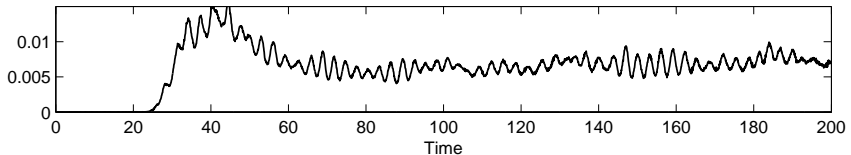

$$
\int_{A} w^{R o}\left\langle\boldsymbol{v}_{H}^{\prime} \cdot \nabla_{H} w^{\prime}\right\rangle_{z} d A
$$

FiguRE 3. The integral quantities on the right hand side of (2.22)-(2.24) for $R o=0.01$. Note first that $\int_{Y} w^{R o} \rho d V=\int_{A} w^{R o}\langle\rho\rangle_{z} d A$ because $w^{R o}$ is independent of the vertical coordinate. Note that the upper most plot is the only one without a definitive sign, and that the values for this exchange are at least one order of magnitude larger than those present in the other plots. 


\subsection{Strong stratification in the presence of weak rotation}

To ensure that the necessary scales are completely resolved in this limit we require that $k_{f} / \mathrm{Fr}$ be smaller than the total number of modes in the simulation (512). This restricts the range of possible Froude numbers, but ensures that all of the small scales introduced by the stratification and forcing are adequately resolved.

The evolution of the potential and kinetic energy is shown in Fig. 4. As in the weak stratification case, there is an initial spin up phase where the potential and kinetic energy both experience a rapid growth, followed by a slowly increasing value of the potential energy and substantial (but less rapid than initially) growth in the kinetic energy. Although there is clearly more energy in the fluctuations than for the part of the flow living on the slow manifold, it is also evident that the growth (in time) of the kinetic energy is due primarily to the increase of kinetic energy on the slow manifold. In contrast, the potential energy on the slow manifold shows little correlation with the total potential energy, implying that there is a substantial amount of fluctuating potential energy in the system. This is not surprising, as the slow dynamics in this limit will exemplify vertically sheared flow (see Embid \& Majda (1998)) wherein the slow density is independent of the horizontal directions and satisfies the one dimensional heat equation in the vertical. Hence, even though these simulations force the density directly the evolution of the flow approximately behaves as one would expect from the asymptotically derived slow dynamics equations.

These observations on the evolution of the energy of the system are strengthened by Fig. 5 which shows the time evolving values for the transfer of energy from the fluctuating part of the flow to the slow manifold. Contrary to the weakly stratified case of the previous subsection, there is no clear evidence for how the energy is exchanged between the slow density and the flow living off the slow manifold, as indicated in the right hand plot of Fig. 5. Again, this physically is reasonable as the bulk of the potential energy lies off the $O(1)$ slow manifold, indicating that the primary exchange between kinetic and potential energy takes place in the fluctuating components of the flow. The relatively small amount of potential energy on the slow manifold does influence the fluctuations and vice versa, but the effect is negligible when compared to the potential energy living off the manifold

as evidenced by the orders of magnitude difference between the upper right hand plot of Fig. 4 and the right hand plot of Fig. 5.

As in the weakly stratified case, the left hand plot of Fig. 5 indicates that the fluctuations act as a source for all time $t>0$ for the kinetic energy on the slow manifold. If such a statement is true for more physically realizable forcing, it indicates that the fluctuating energy acts as a conduit to create kinetic energy on the slow manifold. In a weaker sense this observation was also made by Smith \& Waleffe (2002) where they show that small scale forcing creates the large scale vertically sheared horizontal flows predicted by Embid \& Majda (1998). The impact of this exchange is not as dramatic as in the case of weak stratification and rapid rotation, but does indicate that the components of the flow living off the $O(1)$ slow manifold can act as a source for energy on it.

\subsection{Quasi-geostrophy: simultaneously strong stratification and rapid rotation}

In keeping with the previous two sections, and as opposed to traditional perspectives on quasi-geostrophy we explore the evolution of the kinetic and potential energy on and off of the slow manifold as this limit is approached (i.e. $F r$ and $R o$ are small but finite). Traditionally this limit is studied in the context of the potential vorticity and enstrophy (analog of energy) since the resultant dynamics are greatly simplified in this context. We postpone the investigation of potential enstrophy to a later work allowing a more comprehensive comparison between each of the three limits considered in this paper. 

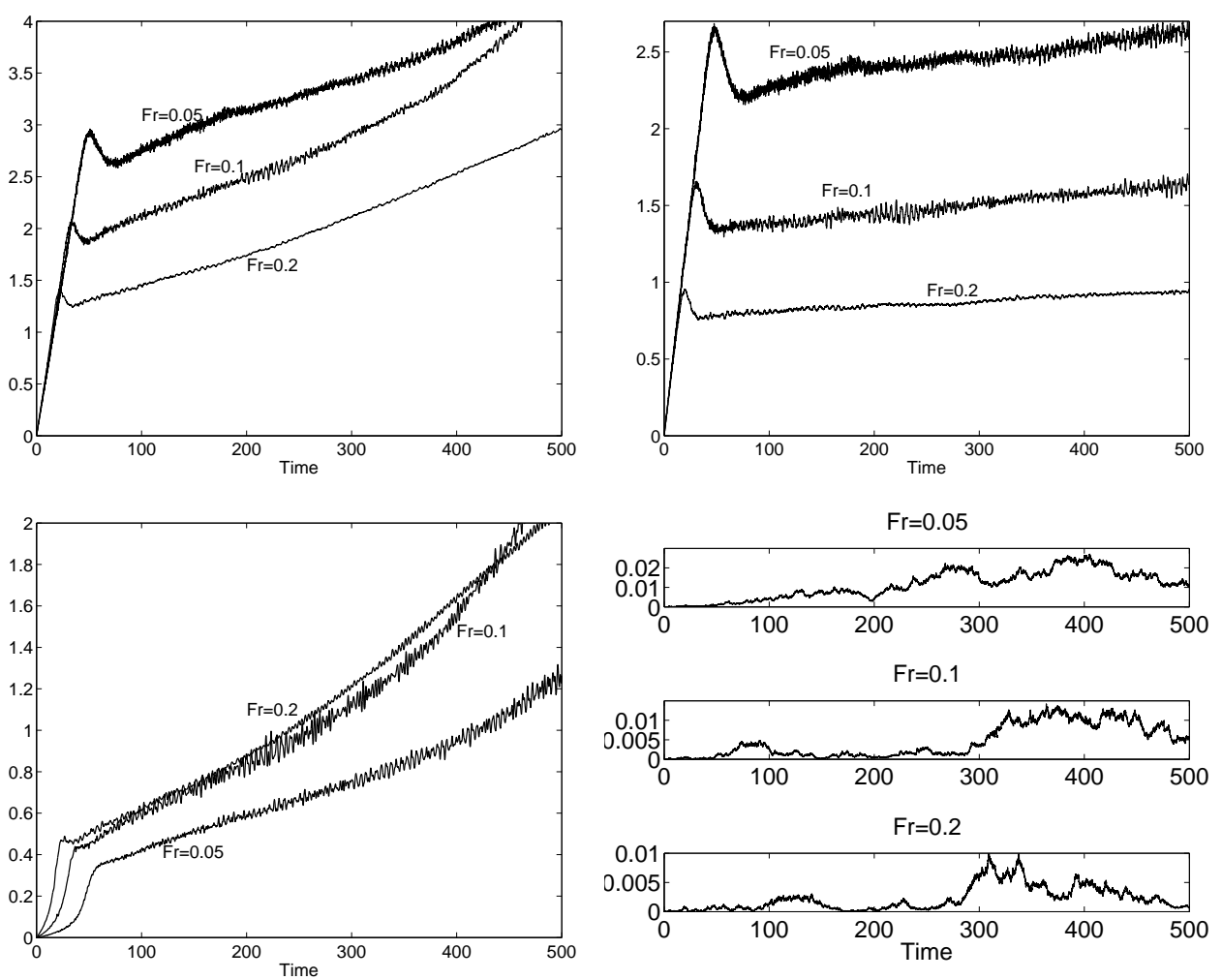

FiguRE 4. The evolution of the kinetic (left) and potential (right) energy. The upper plots are the full energy of the system $\left(\boldsymbol{u}=\boldsymbol{u}^{F r}+\boldsymbol{u}^{\prime}\right)$ and the lower plots are the energy projected onto the slow manifold.
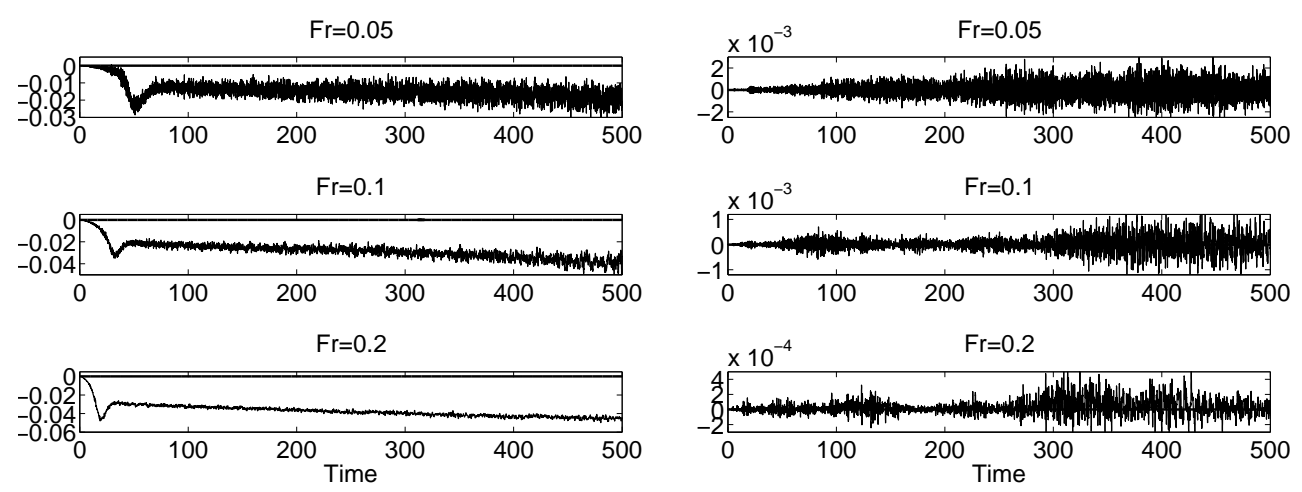

$$
\int_{V} \boldsymbol{v}_{H}^{F r} \cdot\left\{\boldsymbol{v}^{\prime} \cdot \nabla \boldsymbol{v}^{\prime}\right\}_{H} d V
$$

FIGURE 5. The exchange of energy between the non-slow parts of the flow and the kinetic (left) and potential (right) energy on the slow manifold. While the non-slow momentum drives the momentum on the slow manifold, the influence of the density is less clear, i.e. the right hand plots do not exhibit a definitive exchange. 


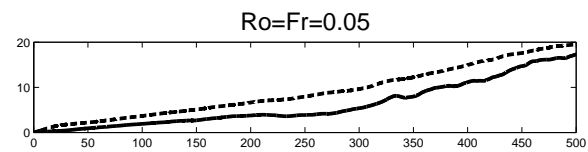

$\mathrm{Ro}=\mathrm{Fr}=0.1$

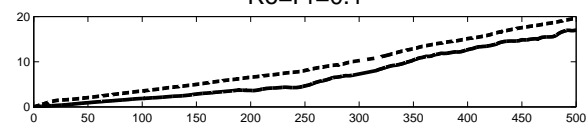

$\mathrm{Ro}=\mathrm{Fr}=0.2$

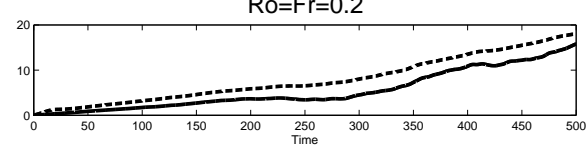

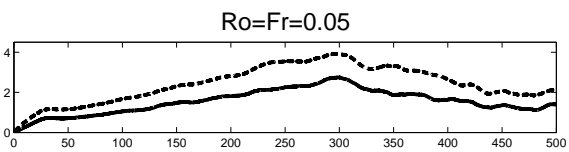

$\mathrm{Ro}=\mathrm{Fr}=0.1$

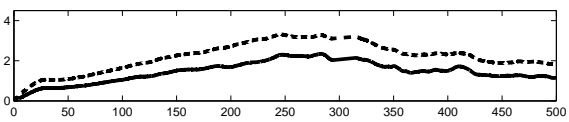

$\mathrm{Ro}=\mathrm{Fr}=0.2$

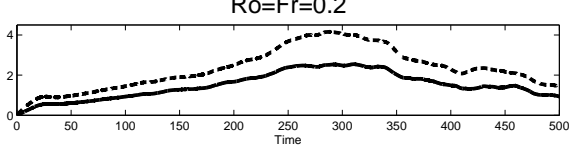

Figure 6. The evolution of the kinetic energy (left) and potential energy (right) for a handful of the rapidly rotating and strongly stratified simulations (quasi-geostrophy). The dashed lines indicate the energy of the full system, while the solid lines is the energy on the slow manifold.

The evolution of the energy for a handful of simulations approaching this limit are shown in Fig. 6. Once again the bulk of the energy lies on the slow manifold, both for kinetic and potential energy, and the energy lying off the slow manifold is quasi-steady, i.e. the gap between the dashed and solid lines is nearly constant. Such a comparison must be taken with caution however, as the projection onto the slow manifold in this limit couples the momentum and density variables. This linear coupling between the dependent variables of the flow to define the slow manifold is indicative of the definition of a potential vorticity variable that will completely describe the evolution via a single dependent variable.

Despite the forcing being applied to the density directly, it is the kinetic energy on the slow manifold that continues growing for all time, whereas the potential energy has an apparent maximum only part way through the simulation. This is consistent with the physical intuition that the system can store only a finite amount of potential energy before it is converted into kinetic energy. The applicability of this statement to both the full system and the flow on the slow manifold indicate the physical vitality of the asymptotic approximation to the reduced system.

As with the other two limits, Figs. 7 and 8 visually reflect how the slow manifold is affected by the fluctuating parts of the flow that lie off it. Fig. 7 displays the integral terms corresponding to the exchange of energy between the fluctuations and the kinetic energy on the slow manifold, and Fig. 8 shows the exchange of energy between the fluctuations and the slow potential energy. Generically these terms do not have an immediately obvious effect on the evolution of the slow manifold. In addition, the conversion of energy from potential to kinetic (as indicated for $R o=F r=0.05$ in the upper plot of Fig. 9) on the slow manifold is at least two orders of magnitude larger than any of the terms shown in Figs. 7 and 8 indicating that the energy on the slow manifold is rapidly shifting between potential and kinetic with relatively minor effects from the non-slow part of the flow.

The fluctuating components of the density variable (advected by the momentum fluctuations) generically contribute to growth in the kinetic and potential energy on the slow manifold (the right hand plots of both Fig. 7 and Fig. 8 are mostly negative). This influence is less significant for the potential energy (see Fig. 8), although such a difference may be explained by the relative values of the total kinetic and potential energies 

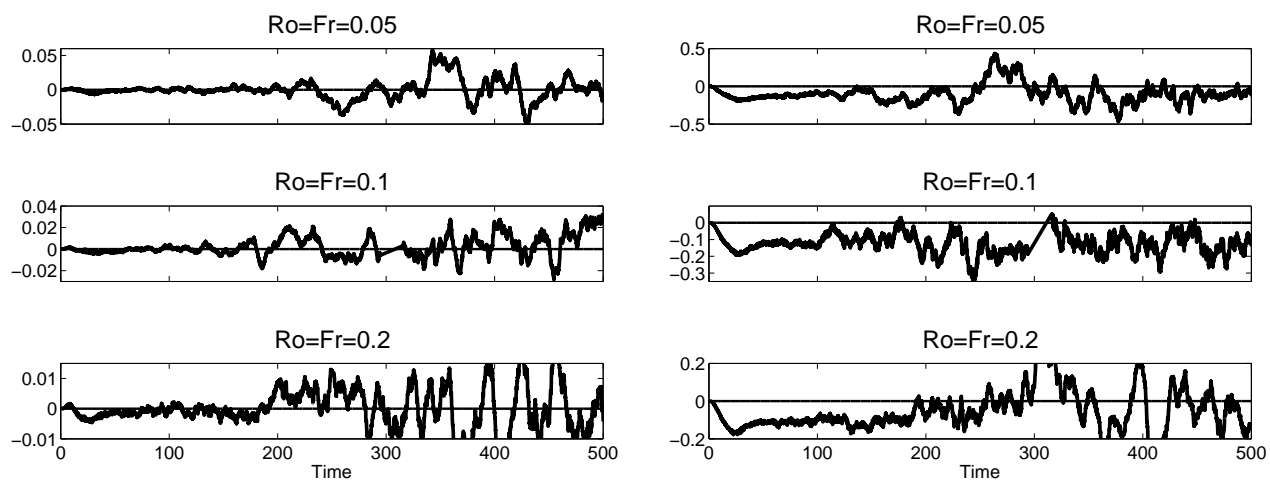

$\int_{V} \boldsymbol{v}_{H}^{Q G} \cdot\left(1-B u^{2} \Delta_{Q G}^{-1} \frac{\partial^{2}}{\partial z^{2}}\right)\left\{\boldsymbol{v}^{\prime} \cdot \nabla \boldsymbol{v}^{\prime}\right\}_{H} d V$

$B u \int_{V} \boldsymbol{v}_{H}^{Q G} \cdot \Delta_{Q G}^{-1}\left[\nabla_{H} \times\left(\hat{z}\left\{\boldsymbol{v}^{\prime} \cdot \nabla \rho^{\prime}\right\}_{z}\right)\right] d V$

FiguRE 7 . The transfer of energy from the fluctuating components of the flow to the slow kinetic energy.

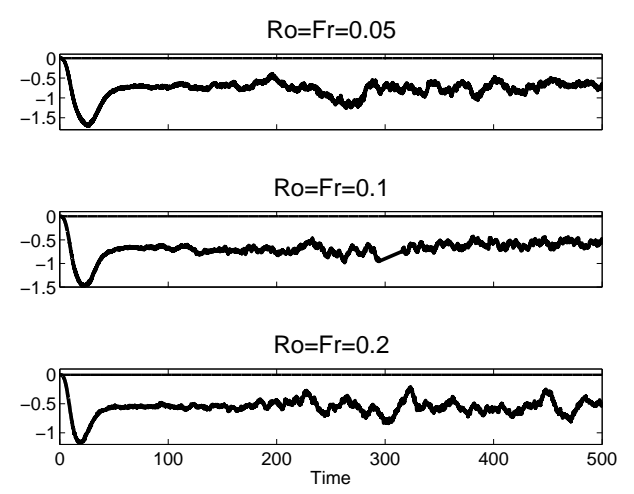

$B u \int_{V} \rho^{Q G} \Delta_{Q G}^{-1} \frac{\partial}{\partial z}\left(\nabla_{H} \times\left\{\boldsymbol{v}^{\prime} \cdot \nabla \boldsymbol{v}^{\prime}\right\}_{H}\right) d V$
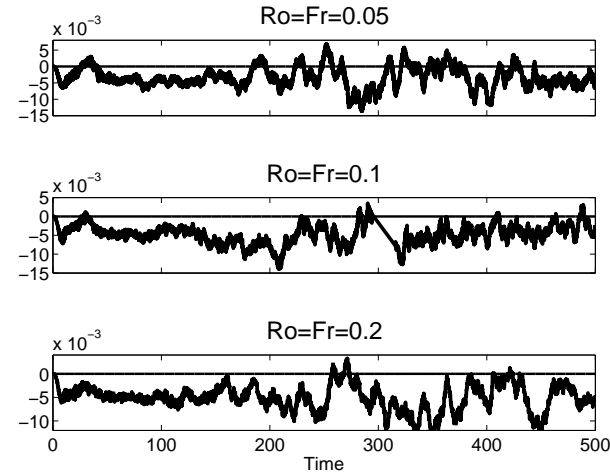

$\int_{V} \rho^{Q G}\left(1-\Delta_{Q G}^{-1} \Delta_{H}\right)\left\{\boldsymbol{v}^{\prime} \cdot \nabla \rho^{\prime}\right\} d V$

Figure 8 . The transfer of energy from the fluctuating components of the flow to the slow potential energy.

(see Fig. 6). The influence of the momentum fluctuations on the slow potential energy is clearly to act as a sink (see left hand plots of Fig. 8), however even a time average value of the effect of these terms on the slow kinetic energy (see left hand plots of Fig. 7) does not reflect an obvious impact on the evolution of the slow energy.

Also, in this limit the relative magnitude of the terms depicted in Figs. 7 and 8 is significant. This is best seen in Fig. 9 where the evolution of each of these terms is depicted for $R o=F r=0.05$. Comparing the magnitude of the exchanges onto and off the slow manifold implies that the density fluctuations have the greatest impact on the slow kinetic energy. The opposite statement holds for the fluctuations in momentum, where the sign of the lower left plot of Fig. 8 indicates that the momentum fluctuations act as a sink for the potential energy on the slow manifold. It appears then that excess slow potential energy is converted not only to slow kinetic, but can also shed off the slow manifold as fluctuating kinetic energy. 


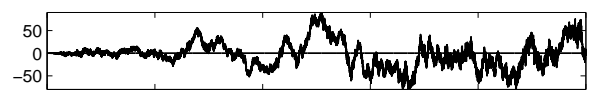

$$
\begin{gathered}
B u \int \rho^{Q G} \Delta_{Q G}^{-1} \frac{\partial}{\partial z}\left(\boldsymbol{v}_{H}^{Q G} \cdot \nabla_{H} \omega^{Q G}\right) d V \\
\int_{V} \boldsymbol{v}_{H}^{Q G} \cdot\left(1-B u^{2} \Delta_{Q G}^{-1} \frac{\partial^{2}}{\partial z^{2}}\right)\left\{\boldsymbol{v}^{\prime} \cdot \nabla \boldsymbol{v}^{\prime}\right\}_{H} d V \\
B u \int_{V} \boldsymbol{v}_{H}^{Q G} \cdot \Delta_{Q G}^{-1}\left[\nabla_{H} \times\left(\hat{z}\left\{\boldsymbol{v}^{\prime} \cdot \nabla \rho^{\prime}\right\}_{z}\right)\right] d V \\
\int_{V} \rho^{Q G}\left(1-\Delta_{Q G}^{-1} \Delta_{H}\right)\left\{\boldsymbol{v}^{\prime} \cdot \nabla \rho^{\prime}\right\} d V \\
B u \int_{V} \rho^{Q G} \Delta_{Q G}^{-1} \frac{\partial}{\partial z}\left(\nabla_{H} \times\left\{\boldsymbol{v}^{\prime} \cdot \nabla \boldsymbol{v}^{\prime}\right\}_{H}\right) d V
\end{gathered}
$$
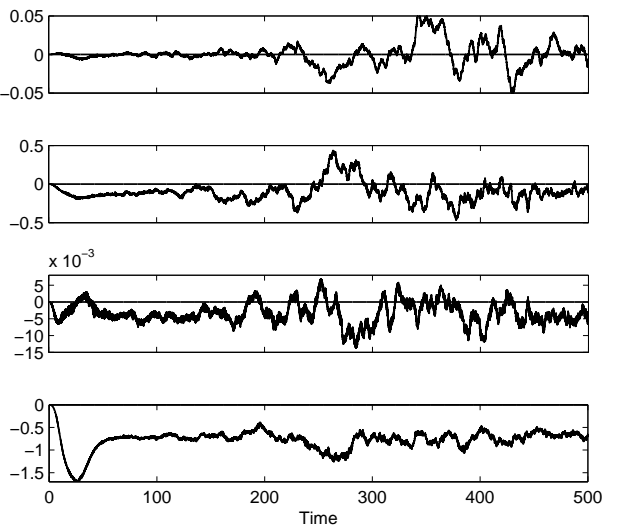

Figure 9. The integral quantities on the right hand side of $(2.33)-(2.34)$ for $R o=F r=0.05$. Note that the term represented in the upper most plot is equivalent (to within a sign) of the first term on the right hand side of (2.33).

\section{The influence of the fluctuating components of the flow on three distinct slow manifolds}

In this paper we have investigated the energy flow onto and off of the slow manifold for the rotating, stratified Boussinesq system of equations that contains three distinctly defined slow manifolds. Using the projection of the flow onto the null space of the fast operator we can decompose the flow into an $O(1)$ slow component and fluctuations about this slow manifold. This allows us to consider the evolution of the slow manifold explicitly both from the perspective of the momentum and density evolution equations, and in terms of kinetic and potential energy.

We performed and discussed numerical simulations that depict the evolution of the slow manifold for each of these three distinct limits. For all three cases and the driving force considered, the energy injected into the system moves onto or stays on the slow manifold (see Figs. 1, 4, and 6) with a quasi-steady amount being left in the fluctuating components of the flow. The primary observation we make is that for all three limiting cases, the growth (in time) in total energy appears to occur primarily on the slow manifold while the fluctuating energy appears to stabilize after an initial transitional state in the simulations. The movement of the energy onto or off the slow manifold can be readily identified for the rapidly rotating and weakly stratified limit, but is less distinct for the other two limiting cases.

As mentioned previously, although the forcing is injecting potential energy, the potential energy cannot grow unbounded, but eventually will be converted into kinetic energy. The evolution equations for the energy on the slow manifold show that only in the QG limit can potential energy convert into kinetic while remaining slow. For the other two limiting cases the excess potential energy must feed into the fluctuating components of the flow. The simulations reported here indicate that a substantial portion of this fluctuating energy is then converted into kinetic energy on the slow manifold.

For the rapidly rotating and weakly stratified, and strongly stratified and weakly ro- 
tating limits, the choice of momentum and density as the prognostic variables leads to an understanding of the evolution of the slow manifold and its interaction with the fluctuating components of the flow. Such clear conclusions and understanding cannot be drawn when quasi-geostrophic balance is of interest. Instead, when the stratification and rotation are both asymptotically strong we observe that the evolution of the slow energy is far more complicated and while it is apparent that the energy in the fluctuations remains bounded, and there is some evidence for how this energy is moved, it isn't as conclusive as for the other two limits. More insights into this limit will likely result by considering a similar analysis and decomposition on the potential vorticity as opposed to the momentum and density.

These results also support ideas used in the analysis of Temam \& Wirosoetisno (2010) where it is shown that the slow manifold (quasi-geostrophy) of the hydrostatic primitive equations is attracting in the sense that after a sufficiently long time the flow will lie within $\sqrt{\epsilon}$ of the slow manifold (this is shown for $O(1)$ definitions of the slow manifold, higher order definitions lead to exponentially close results). As described in Temam \& Wirosoetisno (2011) one of the key elements to this argument is that the fluctuations cannot influence the slow manifold in the infinite limit, something which also holds for each of the three distinct limits considered here (Embid \& Majda 1998; Wingate et al. 2011).

The simulations reported here also highlight a different question that is distinct from the issue of the asymptotic 'stability' of the slow manifold. The simulations shown here indicate that when the forcing is applied indiscriminately to the entire flow (both the slow and fluctuating components), the fluctuating dynamics can play an important role in distributing the energy. It is demonstrated in Farge \& Sadourny (1989); Dewar \& Killworth (1995); Ward \& Dewar (2010) that the $O(1)$ fast waves do not influence the $O(1)$ slow manifold, however the numerical simulations reported here indicate that the higher order waves and other non-slow parts of the flow can play a unique role in determining the evolution of the slow manifold, dependent on which limit is being considered. If the forcing were applied purely on the slow manifold these considerations would not be a concern, but in reality this does not occur in very many circumstances. Thus we may conclude that when there is a force applied to the flow off the slow manifold (as defined here as the null space of the fast operator), it is not sufficient to consider evolution of the flow on this manifold alone, for then the (potentially important) influence of these fluctuating components of the flow would be neglected.

Acknowledgements - We thank T. Haut, A. Farhat, and A. Larios for helpful discussions and relevant insights. This work was supported by the U.S. Department of Energy's LANL/LDRD program.

Appendix: The projection operator for the quasi-geostrophic limit

Here we derive the projection operator for the quasi-geostrophic limit, partially because this limit is distinguishably more complicated, and also the explicit projection operator is not stated in Embid \& Majda (1998), so this derivation not only motivates the derivation of the other 2 limits (similar methods produce the correct results), but also completes the derivations presented in Embid \& Majda (1996, 1998); Wingate et al. (2011).

In order to derive the limiting dynamics for any of the cases considered in this paper, we cast things into spectral space (making use of the Fourier transform on the triply periodic domain considered here). This leads to the Fourier representation of the corresponding 
dependent variables as

$$
\boldsymbol{u}=\sum_{\hat{\boldsymbol{k}}} \hat{\boldsymbol{u}}_{k} e^{\imath \hat{\boldsymbol{k}} \cdot \boldsymbol{x}}
$$

where $\hat{\boldsymbol{k}}=(k, l, m)^{T}$ is the wave number of the Fourier coefficient $\hat{\boldsymbol{u}}_{k}$. This leads to the spectral representation of the linear dispersive operators we are concerned with:

$$
L_{F r} \hat{\boldsymbol{u}}_{k}=\left(\begin{array}{c}
-\frac{k m}{|\hat{\boldsymbol{k}}|^{2}} \hat{\rho}_{k} \\
-\frac{l m}{|\hat{\boldsymbol{k}}|^{2}} \hat{\rho}_{k} \\
\hat{\rho}_{k}-\frac{m^{2}}{|\hat{\boldsymbol{k}}|^{2}} \hat{\rho}_{k} \\
-\hat{w}_{k}
\end{array}\right) \quad L_{R o} \hat{\boldsymbol{u}}_{k}=\left(\begin{array}{c}
-\hat{v}_{k}-\imath \frac{k}{|\hat{\boldsymbol{k}}|^{2}} \hat{\omega}_{k} \\
\hat{u}_{k}-\imath \frac{l}{|\hat{\boldsymbol{k}}|^{2}} \hat{\omega}_{k} \\
-\imath \frac{m}{|\hat{\boldsymbol{k}}|^{2}} \hat{\omega}_{k} \\
0
\end{array}\right)
$$

where

$$
\hat{\omega}_{k}=\imath\left(k \hat{v}_{k}-l \hat{u}_{k}\right)
$$

is the spectral representation of the vertical component of vorticity.

In the quasi-geostrophic limit, both of these operators become asymptotically dominant, so that the full linear operation (in spectral space) is actually given by its matrix representation:

$$
\frac{1}{F r} L_{F r} \hat{\boldsymbol{u}}_{k}+\frac{1}{R o} L_{R o} \hat{\boldsymbol{u}}_{k}=\left(\begin{array}{cccc}
-\frac{1}{R o} \frac{k l}{|\hat{\boldsymbol{k}}|^{2}} & -\frac{1}{R o}\left(1-\frac{k^{2}}{|\hat{\boldsymbol{k}}|^{2}}\right) & 0 & -\frac{1}{F r} \frac{k m}{|\hat{\boldsymbol{k}}|^{2}} \\
\frac{1}{R o}\left(1-\frac{l^{2}}{|\hat{\boldsymbol{k}}|^{2}}\right) & \frac{1}{R o} \frac{k l}{|\hat{\boldsymbol{k}}|^{2}} & 0 & -\frac{1}{F r} \frac{l m}{|\hat{\boldsymbol{k}}|^{2}} \\
-\frac{1}{R o} \frac{l m}{|\hat{\boldsymbol{k}}|^{2}} & \frac{1}{R o} \frac{k m}{|\hat{\boldsymbol{k}}|^{2}} & 0 & \frac{1}{F r}\left(1-\frac{m^{2}}{|\hat{\boldsymbol{k}}|^{2}}\right) \\
0 & 0 & -\frac{1}{F r} & 0
\end{array}\right) \hat{\boldsymbol{u}}_{k} .
$$

To make this representation more amenable to simplification we rewrite the matrix as

$$
A(\hat{\boldsymbol{k}})=\frac{1}{|\hat{\boldsymbol{k}}|^{2}}\left(\begin{array}{cccc}
-\frac{k l}{R o} & -\frac{l^{2}+m^{2}}{R o} & 0 & -\frac{k m}{F r} \\
\frac{k^{2}+m^{2}}{R o} & \frac{k l}{R o} & 0 & -\frac{l m}{F r} \\
-\frac{l m}{R o} & \frac{k m}{R o} & 0 & \frac{k^{2}+l^{2}}{F r} \\
0 & 0 & -\frac{|\hat{\boldsymbol{k}}|^{2}}{F r} & 0
\end{array}\right) .
$$

We need to construct the projection onto the null space of this matrix representation $A(\hat{\boldsymbol{k}})$. To do this, note that the null space of this operator is spanned by the unit vector

$$
\frac{1}{\sqrt{k^{2}+l^{2}+B u^{2} m^{2}}}\left(\begin{array}{c}
l \\
-k \\
0 \\
B u \cdot m
\end{array}\right)
$$

where $B u=F r / R o=O(1)$ is the Burger number, and describes the relative influence of stratification to rotation. In reality we must be concerned with the limits of vanishing horizontal wave number, although it turns out that this limiting case is easily realized from this general construction. Standard linear algebra then allows us to construct the 
projection onto the space spanned by this vector as:

$$
P \hat{\boldsymbol{u}}_{k}=\frac{1}{k^{2}+l^{2}+B u^{2} m^{2}}\left(\begin{array}{c}
l^{2} \hat{u}_{k}-k l \hat{v}_{k}+B u \cdot l m \hat{\rho}_{k} \\
-k l \hat{u}_{k}+k^{2} \hat{v}_{k}-B u \cdot k m \hat{\rho}_{k} \\
0 \\
B u \cdot m\left(l \hat{u}_{k}-k \hat{v}_{k}+B u \cdot m \hat{\rho}_{k}\right)
\end{array}\right) .
$$

Reorganizing terms carefully, and reverting back to real space, this becomes (2.31).

\section{REFERENCES}

Ahlers, G., Grossmann, S. \& Lohse, D. 2009 Heat transfer and large scale dynamics in turbulent Rayleigh-Bénard convection. Review of Modern Physics 81, 503-537.

BAER, F. 1977 Adjustment of initial conditions required to suppress gravity wave oscillations in nonlinear flows. Beitr. Phys. Atmos. 50, 350-366.

BAer, F. \& Tribbia, J. J. 1977 On complete filtering of gravity modes through nonlinear initialization. Monthly Weather Review 105 (12), 1536-1539.

Chandrasekhar, S. 1961 Hydrodynamics and hydro-magnetic stability. Clarendon Press, Oxford, UK.

Charney, J. G. 1947 The dynamics of long waves. Journal of Meteorology 4, 135-162.

Charney, J. G. 1948 On the scale of atmospheric motions. Geophysical Publications 17 (2), 17 pages.

Charney, J. G. 1955 The use of primitive equations of motion in numerical forecasting. Tellus $\mathbf{7}, 22-26$.

Charney, J. G. 1971 Geostrophic tubulence. Journal Of The Atmospheric Sciences 28 (6), 1087.

Charney, J. G., Fjortoft, R. \& von Neumann, J. 1950 Numerical integration of the barotropic vorticity equation. Tellus $\mathbf{2}, 237-254$.

Charney, J. G. \& Phillips, N. A. 1953 Numerical integration of the quasigeostrophic equations for barotropic and simple baroclinic flows. Journal of Meteorology 10, 71-99.

Chasnov, J. R. 1994 Similarity states of passive scalar transport in isotropic turbulence. Physics of Fluids 6, 1036-1051.

Conangla, L., Cuxart, J. \& Solar, M. R. 2008 Characterisation of the nocturnal boundary layer at a site in northern spain. Boundary-Layer Meteorology 128, 255-276.

Coulter, R. L. 1990 A case study of turbulence in the stable nocturnal boundary layer. Boundary-Layer Meteorology 52, 75-91.

Dewar, W. K. \& Killworth, P. D. 1995 Do fast gravity waves interact with geostrophic motion? Deep Sea Research 42 (7), 1063-1081.

EADY, E. T. 1949 Long waves and cyclone waves. Tellus 1 (3), 33-52.

EMBID, P. F. \& MAJDA, A. J. 1996 Averaging over fast gravity waves for geophysical flows with arbitrary potential vorticity. Communications in Partial Differential Equations 21 (3-4), 619-658.

Embid, P. F. \& MAJDA, A. J. 1998 Low Froude number limiting dynamics for stably stratified flow with small or finite Rossby numbers. Geophysical and Astrophysical Fluid Dynamics $87(1-2), 1-50$.

Emery, W. J., Lee, W. G. \& MagaArd, L. 1984 Geographic and seasonal distributions of Brunt-Väisälä frequency and Rossby radii in the north Pacidic and north Atlantic. Journal of Physical Oceanography 14, 294-317.

Farge, M. \& Sadourny, R. 1989 Wave-vortex dynamics in rotating shallow flow. Journal of Fluid Mechanics 206, 433-462.

Ford, R., McIntyre, M. E. \& Norton, W. A. 2000 Balance and the Slow Quasimanifold: Some Explicit Results. Journal of the Atmospheric Sciences 57, 1236-1254.

Heywood, K. J., Garabato, A. C. N. \& Stevens, D. P. 2002 High mixing rates in the abyssal Southern Osean. Nature 415, 1011-1014.

Jones, E. P., Rudels, B. \& Anderson, L. G. 1995 Deep waters of the arctic ocean: origins and circulation. Deep-Sea Research 42, 737-760. 
Julien, K. \& KnOBloch, E. 2007 Reduced models for fluid flows with strong constraints. Journal of Mathematical Physics 48 (065405).

Julien, K., Knobloch, E., Milliff, R. \& Werne, J. 2006 Generalized Quasi-Geostrophy for spatially anisotropic rotationally constrained flows. Journal of Fluid Mechanics 555, $233-274$.

Julien, K., Knobloch, E., Rubio, A. M. \& VAsil, G. M. $2012 a$ Heat Transport in LowRossby-Number Rayleigh-Bénard Convection. Physical Review Letters 109 (254503), 5 pages.

Julien, K., Knobloch, E. \& Werne, J. 1998 A New Class of Equations for Rotationally Constrained Flows. Theoretical and Computational Fluid Dynamics 11, 251-261.

Julien, K., Rubio, A. M., Grooms, I. \& Knobloch, E. $2012 b$ Statistical and physical balances in low Rossby number Rayleigh-Bénard convection. Geophysical and Astrophysical Fluid Dynamics 106 (4-5), 392-428.

Khairoutdinov, M., Randall, D. \& DeMott, C. 2005 Simulations of the Atmospheric General Circulation Using a Cloud-Resolving Model as a Superparameterization of Physical Processes. Journal of the Atmospheric Sciences 62 (7), 2136-2154.

King, E. M., Sellmach, S. \& Aurnou, J. M. 2012 Heat transfer by rapidly rotating rayleighbénard convection. Journal of Fluid Mechanics 691, 568-582.

Klainerman, S. \& Majda, A. J. 1981 Singular limits of quasilinear hyperbolic systems with large parameters and the incompressible limit of compressible fluids. Communications in Pure and Applied Mathematics 34 (4), 481-524.

Kreiss, H.-O. \& Lorenz, J. 1994 On the existence of slow manifolds for problems with different timescales. Philosophical Transactions: Physical Science and Engineering 346, 159-171.

Kumar, M. S., Anandan, V. K., RaO, T. N. \& Reddy, P. N. 2012 A climatological study of the nocturnal boundary layer over a complex-terrain station. Journal of Applied Meteorology and Climatology $\mathbf{5 1}$ (4), 813-825.

LeIth, C. E. 1980 Nonlinear Normal Mode Initialization and Quasi-Geostrophic Theory. Journal of the Atmospheric Sciences 37, 958-968.

LELONG, P. \& Riley, J. J. 1991 Internal wave-vortical mode interactions in strongly stratified flows. Journal of Fluid Mechanics 232, 1-19.

LiU, Y. \& EckE, R. E. 2009 Heat transport measurements in turbulent rotating rayleigh-bénard convection. Physical Review E $\mathbf{8 0}$ (036314), 12 pages.

Lorenz, E. N 1980 Attractor Sets and Quasi-Geostrophic Equilibrium. Journal of the Atmospheric Sciences 37, 1685-1699.

Lorenz, E. N. 1986 On the Existence of a Slow Manifold. Journal of the Atmospheric Sciences 43 (15), 1547-1557.

Lorenz, E. N. \& Krishnamurthy, V. 1987 On the Nonexistence of a Slow Manifold. Journal of the Atmospheric Sciences 44 (20), 2940-2950.

MachenAuer, B. 1977 On the dynamics of gravity oscillations in a shallow water model, with application to normal mode initialization. Beitrge zur Physik der Atmosphre 50, 253-271.

Mahrt, L., Sun, J., Blumen, W., Delany, T. \& Oncley, S. 1998 Nocturnal boundary-layer regimes. Boundary-Layer Meteorology 88, 255-278.

Majda, A. 1984 Compressible Fluid Flow and Systems of Conservation Laws in Several Space variables. Applied Mathematics Series . Springer-Verlag, New York, New York.

Majda, AJ \& Grote, MJ 1997 Model dynamics and vertical collapse in decaying strongly stratified flows. Physics of fluids 9 (10), 2932-2940.

Pedlosky, J. 1987 Geophysical Fluid Dynamics. Springer-Verlag.

Riley, J. J. \& LELONG, M. P. 2000 Fluid motions in the presence of strong stable stratification. Annual Review of Fluid Mechanics 32, 613-657.

Saujani, S. \& Shepherd, T. G. 2002 Comments on "Balance and the Slow Quasimanifold: Some Explicit Results". Journal of the Atmospheric Sciences 59, 2874-2877.

Schochet, S. 1987 Singular limits in bounded domains for quasilinear symmetric hyperbolic systems having a vorticity equation. Journal of Differential Equations 68 (3), 400-428.

Schochet, S. 1994 Fast singular limits of hyperbolic pde's. Journal of Differential Equations 114, 476-512.

Smith, L. M. \& Waleffe, F. 1999 Transfer of energy to two-dimensional large scales in forced, rotating three-dimensional turbulence. Physics of Fluids 11 (6), 1608-1622. 
Smith, L. M. \& WAleffe, F. 2002 Generation of slow large scales in forced rotating stratified turbulence. Journal of Fluid Mechanics 451, 145-168.

Sprague, M., Julien, K., Knobloch, E. \& Werne, J. 2006 Numerical simulation of an asymptotically reduced system for rotationally constrained convection. Journal of Fluid Mechanics 551, 141-174.

Staniforth, A. \& Wood, N. 2008 Aspects of the dynamical core of a nonhydrostatic, deepatmosphere, unified weather and climate-prediction model. J. Comput. Phys. 227 (7), 34453464.

Stone, P.H., Hess, S., Hadlock, R. \& Ray, P. 1969 Preliminary results of experiments with symmetric baroclinic instabilities. J Atmos Sci 26 (5), 991 - 6.

Stone, P. H. 1966 On non-geostrophic baroclinic stability. Journal of the Atmospheric Sciences 45, $390-400$.

Stone, P. H. 1971 Baroclinic stability under non-hydrostatic conditions. Journal of Fluid Mechanics pp. 659-671.

Stone, P. H. 1972 On non-geostrophic baroclinic stability. III. The momentum and heat transports. Journal of the Atmospheric Sciences 29 (3), 419 - 26.

Sukhatme, Jai, Majda, Andrew J. \& Smith, Leslie M. 2012 Two-dimensional moist stratified turbulence and the emergence of vertically sheared horizontal flows. Physics of Fluids 24 (3), 036602.

Temam, R. \& Wirosoetisno, D. 2011 Slow Manifolds and Invariant Sets of the Primitive Equations. Journal of the Atmospheric Sciences 68, 675-682.

Temam, R. M. \& Wirosoetisno, D. 2010 Stability of the slow manifold in the primitive equations. SIAM Journal of Mathematical Analysis 42 (1), 427-458.

Timmermans, M. L. 2010 Personal Communication.

Timmermans, M. L., Melling, H. \& Rainville, L. 2007 Dynamics in the deep Canada Basin, Arctic Ocean, inferred by thermistor-chain time series. Journal of Physical Oceanography 37, 1066-1076.

TribBiA, J. J. 1979 Nonlinear initialization on an equatorial beta-plane. Monthly Weather Review 107, 704-713.

Vallis, G. K. 2006 Atmospheric and Oceanic Fluid Dynamics: Fundamentals and Large-Scale Circulation. Cambridge University Press, 745 pp.

VAn Haren, H. \& Millot, C. 2005 a Gyroscopic waves in the Mediterranean sea. Geophysical Research Letters 32 (24), 1-4.

Van Haren, H. \& Millot, C. $2005 b$ Rectilinear and circular inertial motions in the western mediterranean sea. Deep-Sea Research I 51, 1441-1455.

Vanneste, J. 2013 Balance and Spontaneous Wave Generation in Geophysical Flows. Annual Review of Fluid Mechanics 45, 145-172.

VAnneste, J. \& Yavneh, I. 2004 Exponentially Small Inertia-Grarity Waves and the Breakdown of Quasigeostrophic Balance. Journal of the Atmospheric Sciences 61 (2), 211-223.

WARD, M. L. \& Dewar, W. K. 2010 Scattering of gravity waves by potential vorticity in a shallow-water fluid. Journal of Fluid Mechanics 663, 478-506.

WARn, T. 1986 Statistical mechanical equilibria of the shallow-water equations. Tellus A 38, $1-11$.

WARn, T. 1997 Nonlinear balance and quasi-geostrophic sets. Atmosphere-Ocean 35, 135-145.

WARN, T. \& MÉNARD, R 1986 Nonlinear balance and gravity-intertial wave saturation in a simple atmospheric model. Tellus 38A, 285-294.

Werner, S. R., Beardsley, R. C., Lentz, S. J., Hebert, D. L. \& Oakey, N. S. 2003 Observations and modeling of the tidal bottom boundary layer on the southern flank of georges bank. Journal of Geophysical Research: Oceans 108 (C11), 6/1.

Wingate, B. A., Embid, P., Holmes-Cerfon, M. \& Taylor, M. A. 2011 Low Rossby limiting dynamics for stably stratified flow with finite Froude number. Journal of Fluid Mechanics 676, 546-571.

Zhong, J.-Q. \& Ahlers, G. 2010 Heat transport and the large-scale circulation in rotating turbulent rayleigh-bénard convection. Journal of Fluid Mechanics 665, 300-333. 